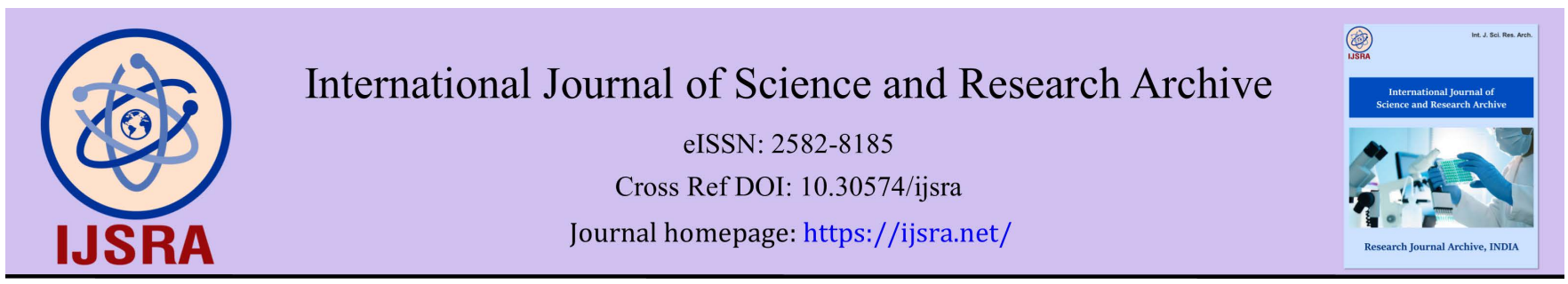

(RESEARCH ARTiClE)

\title{
Formulation and evaluation of atorvastatin calcium niosomes
}

\author{
Neha Rani ${ }^{1}$, Rupali Rana ${ }^{1}$, Reena Thakur ${ }^{1}$, Shivali Singla ${ }^{1, *}$ and Sachin Goyal ${ }^{2}$ \\ ${ }^{1}$ Department of Pharmaceutics, Himalayan Institute of Pharmacy, Kala-Amb, H.P., India. \\ 2 Department of Phamacology, Himalayan Institute of Pharmacy, Kala-Amb, H.P., India.
}

International Journal of Science and Research Archive, 2021, 02(01), 116-124

Publication history: Received on 13 January 2021; revised on 15 February 2021; accepted on 17 February 2021

Article DOI: https://doi.org/10.30574/ijsra.2021.2.1.0028

\begin{abstract}
Atorvastatin calcium is a HMG-CoA reductase inhibitor used for the treatment of hyperlipidaemia. It has oral bioavailability of $\leq 12 \%$. It also undergoes high first pass metabolism. It is highly soluble in acidic $\mathrm{pH}$ and absorbed more in the upper part of the gastrointestinal tract. In order, to improve the absorption and its oral bioavailability, niosomes of Atorvastatin calcium have been formulated and evaluated on different parameters. Niosomes play an increasingly important role in drug delivery as they can reduce toxicity and 000000000 modify pharmacokinetic and bio-availability. Niosomes formulations of Atorvastatin calcium were successfully developed by thin film hydration technique using nonionic surfactant i.e. Span 40, Span 60 Span 80, Tween 20, Tween 40, Tween 80 and cholesterol at different concentrations. The formulations were evaluated for size, shape, and entrapment efficiency. In-vitro release and stability studies also performed. Results indicated that Niosomes were prepared succesfully work as promising drug carriers and promising drug delivery module.
\end{abstract}

Keywords: Atorvastatin calcium; Niosomes; Surfactants; Thin film hydration technique; In-vitro release, Stability studies

\section{Introduction}

Drug delivery systems using colloidal particulate carriers such as liposomes or niosomes have distinct advantages over conventional dosage forms because the particles can act as drug-containing reservoirs. [1] The rationale of (NDDS) novel drug delivery system is to provide a therapeutic amount of drug to reach to the site of action and then preserve the most wanted drug concentration to produce therapeutic effect. The drug- delivery system should deliver drug at a rate control by the necessarily of the body during a specific duration of treatment. [2] The use of non-ionic surfactant vesicles (niosomes) as drug carrier systems has distinct advantages over conventional dosage.[3] The different structure of niosome presents an effective novel drug delivery system with ability of loading both hydrophilic and lipophilic drugs. They can increase the drug efficacy, reduce drug side effects, increase the drug solubility, and develop an effective topical delivery.[4] The rationale of (NDDS) novel drug delivery system is to provide a therapeutic amount of drug to reach to the site of action and then preserve the most wanted drug concentration to produce therapeutic effect. The drug- delivery system should deliver drug at a rate control by the necessarily of the body during a specific duration of treatment [5]. The use of non-ionic surfactant vesicles (niosomes) as drug carrier systems has distinct advantages over conventional dosage. [6] They can increase the drug efficacy, reduce drug side effects, increase the drug solubility, and develop an effective topical delivery [7]. Niosomes are biodegradable, biocompatible non-immunogenic and exhibit flexibility in their structural characterization. And they has been broadly evaluated for controlled release and targeted delivery for the treatment of cancer, viral infections and other microbial diseases. Niosomes serve as drug depot in the body which releases the drug in a controlled manner through its bilayer providing sustained release of the enclosed drug [8]. These days, Niosomes are gaining more fame because of their stability, ease of preparation, increasing drug

\footnotetext{
* Corresponding author: Shivali Singla

Himalayan Institute of Pharmacy, Kala-Amb.
}

Copyright (C) 2021 Author(s) retain the copyright of this article. This article is published under the terms of the Creative Commons Attribution Liscense 4.0. 
efficacy, achiving reduced toxicity, and most importantly their site targeted action. These are significant for drug targeting, particularly in chemotherapy, wherein, the restrictive factor is the traditional lack of selectivity of drugs towards cancer cells and tissues. Niosomes can entrap both hydrophilic and lipophilic drugs and prolong the circulation of entrapped drug [9].

Atorvastatin calcium (ATC) is the most ideal molecule among statins, used to treat moderate to severe hypercholesterolemia, it has oral bioavailability of less than $12 \%$. It also undergoes high first pass metabolism. It is highly soluble in acidic $\mathrm{pH}$ and absorbed more in the upper part of the GIT. Atorvastatin is used for the lowering of serum cholesterol level. According to biopharmaceutical classification, it belongs to Class-II drug. It has very less solubility $(<1 \mathrm{mg} / \mathrm{ml})$, which limits its release from the formulation. It is mostly taken by the liver which results in low systemic bioavailability (14\%) due to the first pass metabolism by the liver. In order, to improve the absorption and its oral bioavailability, niosomes of Atorvastatin calcium have been formulated and evaluated on different parameters [10].

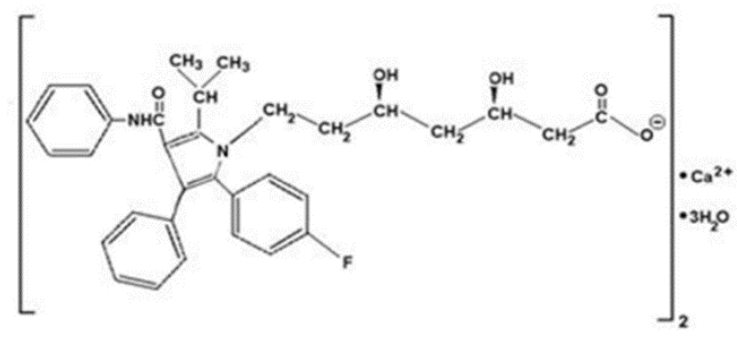

Figure 1 Structure of Atorvastatin calcium

\section{Material and methods}

Atorvastatin calcium was a gift from Lupin India Ltd. Span 40, Span 60 Span 80 and Tween 20, Tween40, Tween 60 obtained from Qualikems Fine Chem Pvt. Ltd. Varodara, India., Cholesterol, Octanol from Loba Chem. Pvt ltd, Mumbai, India. All the chemicals were of analytical grade

\subsection{Formulation development by Thin Film Hydration Technique [11]}

Niosomes containing Atorvastatin calcium were prepared by thin film hydration method using a lipid mixture consisting of surfactant (span 40, span 60, span 80 and tween 20, tween40, tween 60) and Cholesterol, at different specified ratios as given in Table 1. Drug, Surfactant and Cholesterol were dissolved in $10 \mathrm{ml}$ of chloroform. The lipid mixture was then transferred to a $100 \mathrm{ml}$ round bottom flask, and the solvent was evaporated under reduced pressure at a temperature of $55-65^{\circ} \mathrm{C}$, using a rotary evaporator until the formation of a thin lipid film. The formed film was hydrated with $20 \mathrm{ml}$ of Phosphate buffer saline $\mathrm{pH} 7.4$. The hydration was continued for $1 \mathrm{~h}$, while the flask was kept rotating at $55-65^{\circ} \mathrm{C}$ in the rotary evaporator [12]. The hydrated niosomes containing entrapped drugs of varying size were obtained.

Table 1 Compositions of the Atorvastatin calcium Niosomes formulations

\begin{tabular}{|l|l|l|l|}
\hline S.No. & Formulation Code & Surfactant & Drug:Surfactant:Cholesterol (mg) \\
\hline 1. & ANS1 & Span 40 & $100: 100: 100$ \\
\hline 2. & ANS2 & Span 40 & $100: 200: 100$ \\
\hline 3. & ANS3 & Span 60 & $100: 100: 100$ \\
\hline 4. & ANS4 & Span 60 & $100: 200: 100$ \\
\hline 5. & ANS5 & Span 80 & $100: 100: 100$ \\
\hline 6. & ANS6 & Span 80 & $100: 200: 100$ \\
\hline 7. & ANS7 & Tween 20 & $100: 100: 100$ \\
\hline 8. & ANS 8 & Tween 20 & $100: 200: 100$ \\
\hline 9. & ANS9 & Tween 40 & $100: 100: 100$ \\
\hline 10. & ANS10 & Tween 40 & $100: 200: 100$ \\
\hline 11. & ANS11 & Tween 60 & $100: 100: 100$ \\
\hline 12. & ANS12 & Tween 60 & $100: 200: 100$ \\
\hline
\end{tabular}




\section{Evaluation of prepared niosomes}

\subsection{Particle size [13]}

Vesicle size determination was carried out using an optical microscopy with a calibrated eyepiece micrometer. About 200 niosomes were measured individually, average was taken, and their size range, mean diameter were calculated.

\subsection{Entrapment efficiency [14]}

The percentage entrapment efficiency of the vesicles was determined by freeze thawing centrifugation technique. Niosomal suspension was filled in drop tubes and stored at $-20^{\circ} \mathrm{C}$ in a refrigerator for 24 hours. After 24 hours niosomal suspension was taken from refrigerator and stored at room temperature. The niosomal suspension was centrifuged at 1500 X G rpm for 30 minute. Supernatant containing unentrapped drug was withdrawn and diluted with water methanol mixture (80:20), then measured UV spectrophotometrically at $244 \mathrm{~nm}$ against water methanol mixture as standard. Entrapment efficiency (EE) was calculated.

\subsection{Scanning Electron Microscopy [15]}

The shape and surface characteristics of niosomes were evaluated by Scanning Electron Microscopy (SEM). The lyophilized sample was mounted directly on to the sample holder using double-sided sticking tape and after gold coating images were recorded at the required magnification.

\subsection{In-vitro drug release [16]}

The in-vitro permeation of Atorvastatin from niosomal formulation was studied using locally fabricated diffusion cell. The in-vitro diffusion of the drug through egg membrane was performed. It was clamped carefully to one end of the hollow glass tube of $17 \mathrm{~mm}$ (area $2.011 \mathrm{~cm}^{2}$ ) (dialysis cell) this acted as donor compartment. 100 ml of phosphate buffer saline PBS 7.4 was taken in a beaker which was used as a receptor compartment. The known quantity was spread uniformly on the membrane. The donor compartment was kept in contact with the receptor compartment and the temperature was maintained at $37 \pm 0.1^{\circ} \mathrm{C}$. The solutions of the receptor side were stirred by externally driven Tefloncoated magnetic bars. At predetermined time intervals, sample was withdrawn and replaced by $4 \mathrm{ml}$ of PBS. The drug concentrations in the aliquot were determined at $244 \mathrm{~nm}$ against appropriate blank. This experiment was done in triplicate and average value was reported. In- vitro permeation studies were conducted for different formulation and effect of variation in composition on permeation rate. Drug release data was normalized by converting the drug concentrations in solution to a percentage of cumulative drug release and was shown graphically.

\subsection{Accelerated Stability study $[17,18]$}

Stability studies carried out by storing the prepared niosomes of Atorvastatin calcium at a range of temperature conditions like room temperature $(25 \pm 0.50 \mathrm{C})$, refrigeration on $(2-80 \mathrm{C})$, and elevated temperature $(45 \pm 0.50 \mathrm{C})$ for a period of 12 weeks. In which Drug content and variation in the average vesicle diameter were periodically monitored. ICH (International Conference on Harmonisation) guidelines suggests stability studies for dry niosomes powder meant for reconstitution should be studied for accelerated stability at $75 \%$ relative humidity as per international climatic zones and climatic conditions.

\section{Results and discussion}

A successful challenge was made to formulate twelve formulations of Atorvastatin calcium niosomes formulations by Thin film hydration technique using nonionic surfactant and cholesterol at different concentrations. The pure drug shows sensitivity to light and moisture. Therefore formulating it into niosomes can solve this problem to a large extent. The mean particle diameter of the Atorvastatin calcium niosomes was between 2.13-2.69 $\mu \mathrm{m}$ for all twelve formulations. Particles of all formulations were smooth, oval and discrete. The entrapment efficiency of the niosomes was between 66.32-89.42\% The entrapment efficiency was found to be higher with the batch ANS12 (89.42\%), which may have an optimum surfactant cholesterol ratio to provide a high entrapment of Atorvastatin calcium. The higher entrapment may be explained by high cholesterol content ( $\sim 50 \%$ of the total lipid). It was also observed that very high cholesterol content had a lowering effect on drug entrapment to the vesicles. This could be due to the fact that cholesterol beyond a certain level starts disrupting the regular bi-layered structure leading to loss of drug entrapment. 
Table 2 Properties of Atorvastatin calcium Niosomes

\begin{tabular}{|l|l|l|l|l|}
\hline S.N. & $\begin{array}{l}\text { Formulation } \\
\text { Code }\end{array}$ & $\begin{array}{l}\text { Average mean diameter of } \\
\text { nonsonicated niosomes }(\boldsymbol{\mu m})\end{array}$ & $\begin{array}{l}\text { \% Entrapment } \\
\text { efficacy }\end{array}$ & $\begin{array}{l}\text { Viscosity } \\
\text { (Centipoise) }\end{array}$ \\
\hline 1. & ANS1 & $2.43 \pm 0.11$ & $66.32 \pm 0.11$ & 2.016 \\
\hline 2. & ANS2 & $2.46 \pm 0.15$ & $68.31 \pm 0.33$ & 2.573 \\
\hline 3. & ANS3 & $2.51 \pm 0.19$ & $71.12 \pm 0.19$ & 2.157 \\
\hline 4. & ANS4 & $2.33 \pm 0.11$ & $81.32 \pm 0.13$ & 3.322 \\
\hline 5. & ANS5 & $2.15 \pm 0.32$ & $84.41 \pm 0.41$ & 2.336 \\
\hline 6. & ANS6 & $2.33 \pm 0.15$ & $72.45 \pm 0.15$ & 2.520 \\
\hline 7. & ANS7 & $2.47 \pm 0.22$ & $71.42 \pm 0.09$ & 2.167 \\
\hline 8. & ANS 8 & $2.69 \pm 0.31$ & $85.37 \pm 0.12$ & 3.634 \\
\hline 9. & ANS9 & $2.33 \pm 0.08$ & $68.51 \pm 0.08$ & 2.349 \\
\hline 10. & ANS10 & $2.50 \pm 0.06$ & $67.42 \pm 0.21$ & 1.686 \\
\hline 11. & ANS11 & $2.13 \pm 0.11$ & $68.12 \pm 0.08$ & 2.768 \\
\hline 12. & ANS12 & $2.44 \pm 0.14$ & $89.42 \pm 0.19$ & 3.814 \\
\hline
\end{tabular}

\subsection{Scanning Electron Microscopy}

Morphology and internal cross-sectional structure of the microspheres were investigated with a scanning electron microscope. SEM is one of the common methods used owing to the simplicity of sample preparation and ease of operation. Scanning electron photomicrographs of the selected formulation shown in figure 2. SEM indicates that the niosomes were spherical with a smooth surface; distinct pores were evident on the surface of niosomes, which will be responsible for the release. The photomicrographs also showed presence of loose crystals of drug on the surface of a few niosomes.

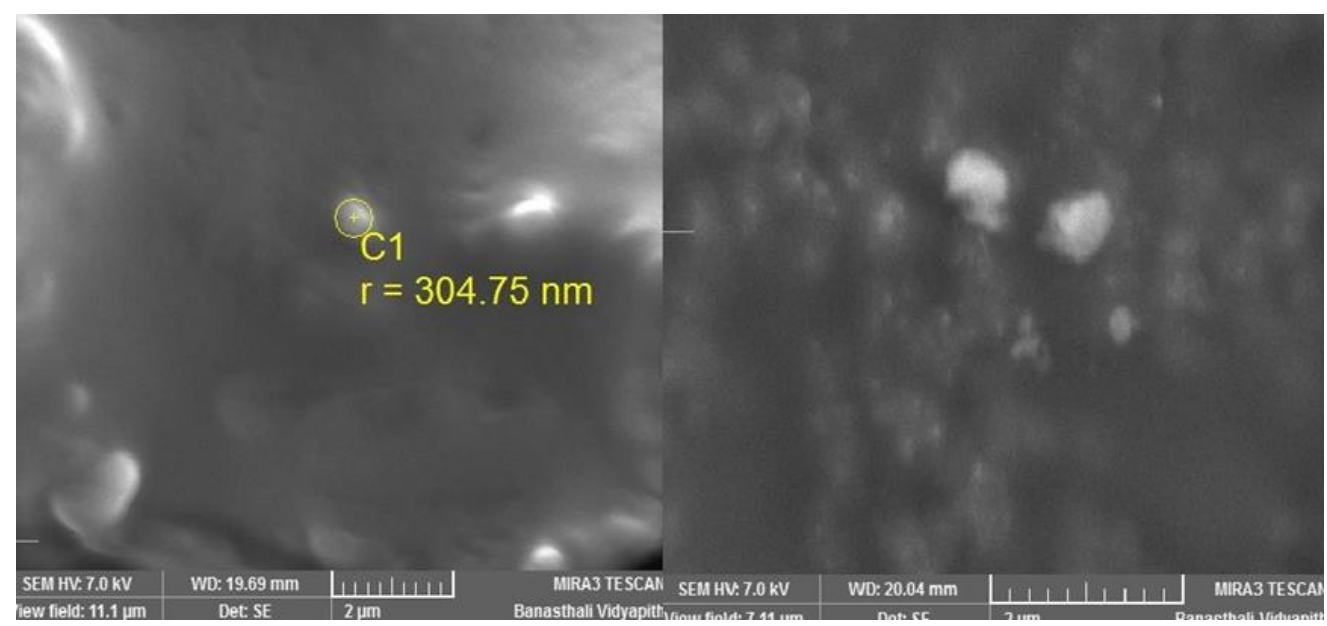

Figure 2 Photograph of Atorvastatin calcium niosomes of batch ANS4 and ANS9

\subsection{In-vitro drug releasestudy}

The in-vitro permeation of Atorvastatin calcium from niosomal formulation was studied using locally fabricated diffusion cell. The cumulative percent drug release after $11 \mathrm{hrs}$ of the Atorvastatin calcium niosomes in between 32.46-85.43\%. The formulation containing tween showed less permeation compared with the preparation containing span. It might be due to the larger size of the vesicles and less lipophilic nature of the Tween, which makes it more difficult for these vesicles to penetrate or fuse with skin whereas, the inclusion of span which is more lipophilic than tween further increased the lipophilicity of the drug leading to better penetration. Rapid drug leakage was observed during the initial phase. However, after that a slow release occurred. This could be because 
the drug is mainly incorporated between the fatty acid chains in the lipid bilayer of niosomal vesicles which leads to rapid ionization and release upon dispersing niosomes in large buffer $(\mathrm{pH} 7.4)$ volumes until reaching equilibrium.

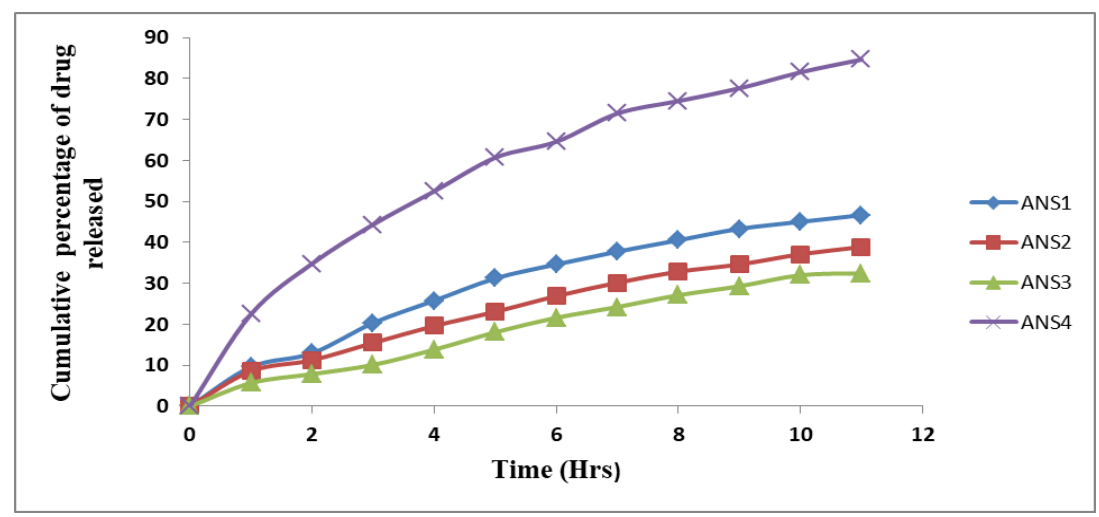

Figure 3 Percentage of drug released from batch ANS1 to ANS4

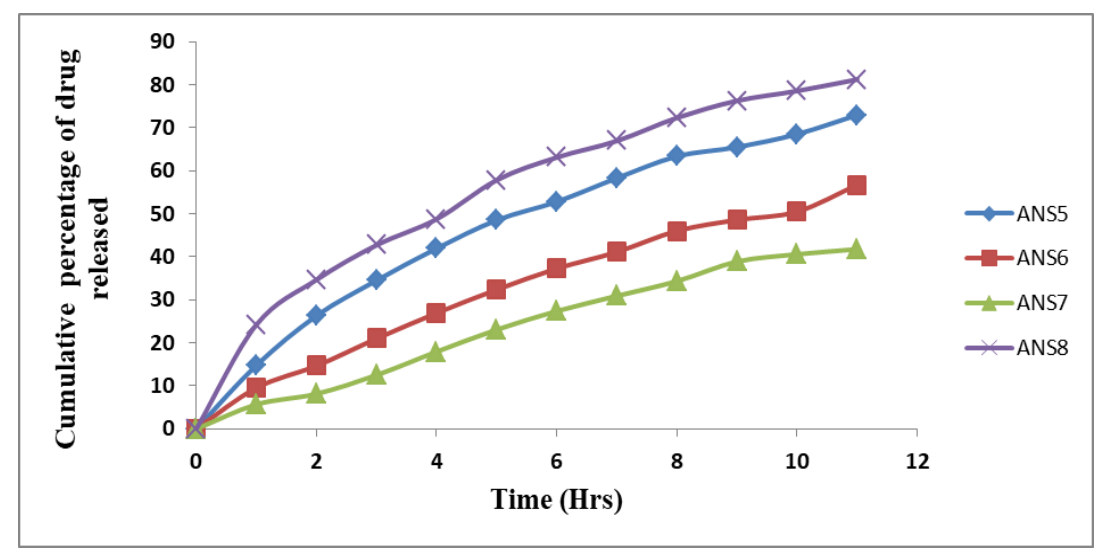

Figure 4 Percentage of drug released from batch ANS5to ANS8

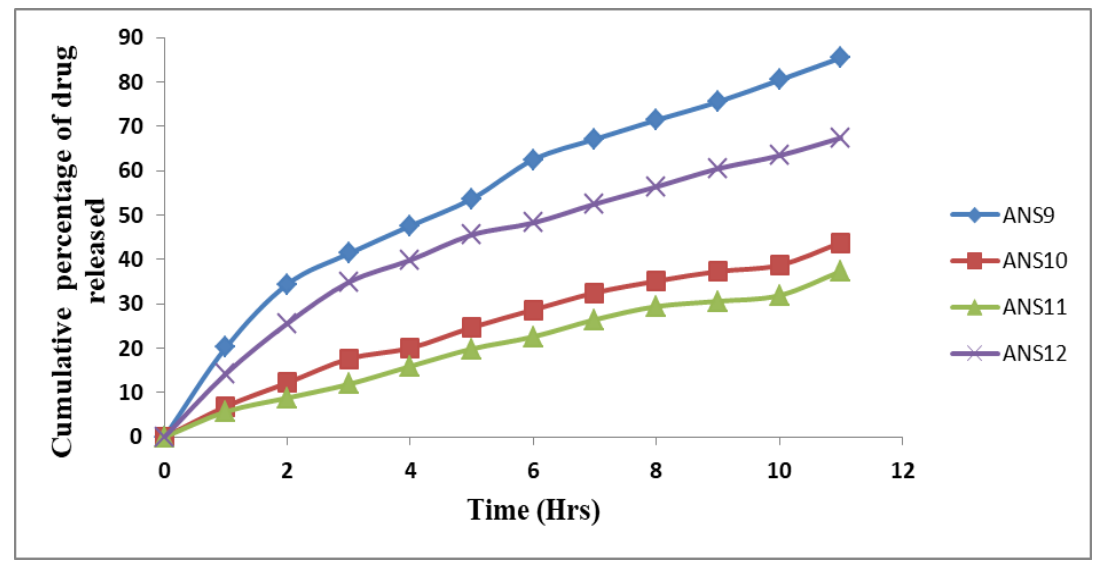

Figure 5 Percentage of drug released from batch ANS9 to ANS12

\subsection{Kinetic modeling for Niosomes Formulation ANS9}

Different kinetic model for in-vitro release study niosomes of Atorvastatin calcium formulation ANS9 are shown in fig. 6-9 respectively. The results obtained in the in-vitro drug release studies were plotted in four models i.e. zero order kinetic model, first order kinetic model, Higuchi kinetic model, and Korsmeyer-peppas release model. 
This indicates that the drug release is controlled by diffusion of the drug through the pores. The formulations are best fitted into the Peppas model. The ' $n$ ' values of these indicate that in these formulations followed Fickian controlled release mechanism and in addition, the release appears to be also by erosion and is drug - dissolution limited.

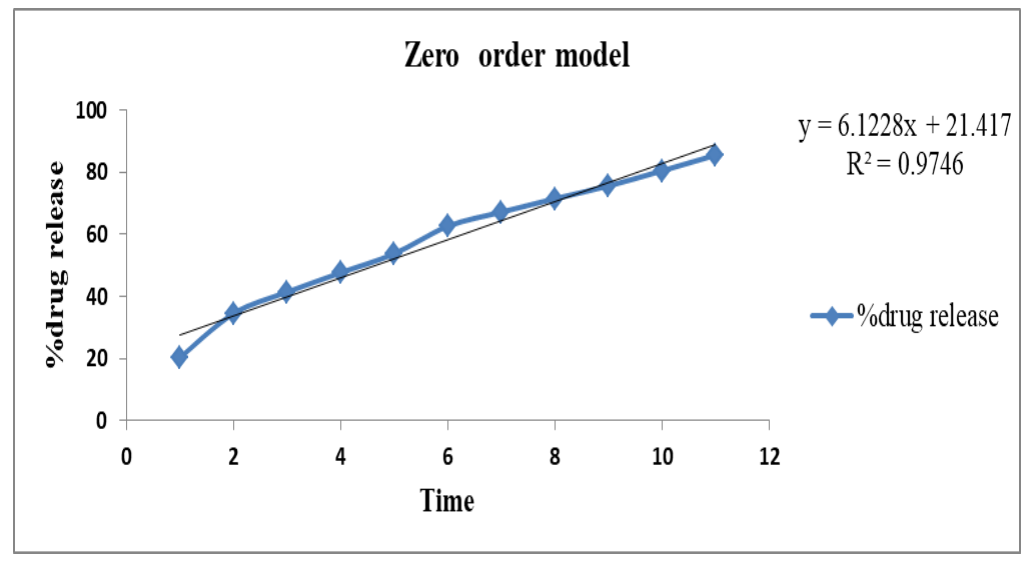

Figure 6 Zero order release model

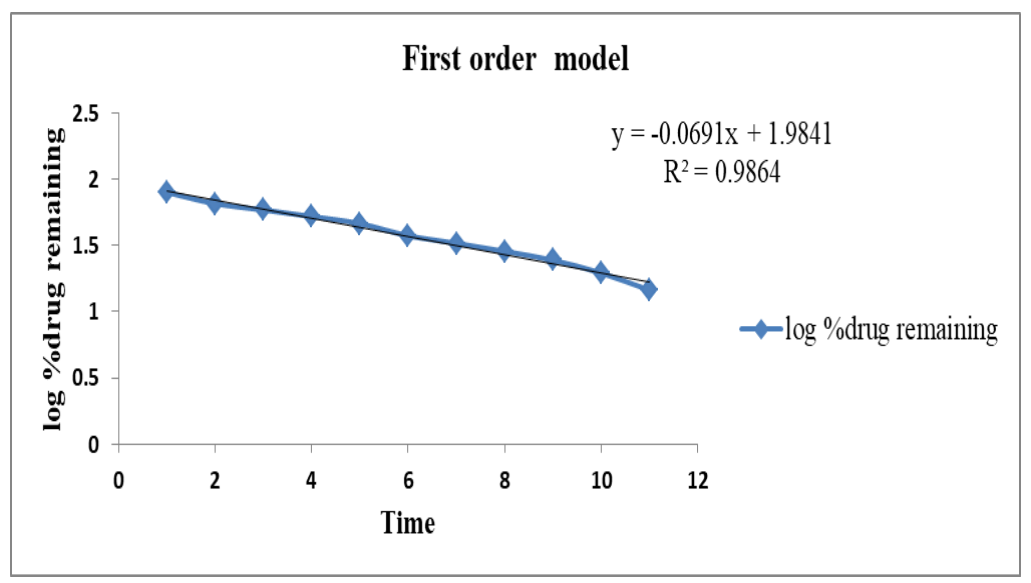

Figure 7 First order release model

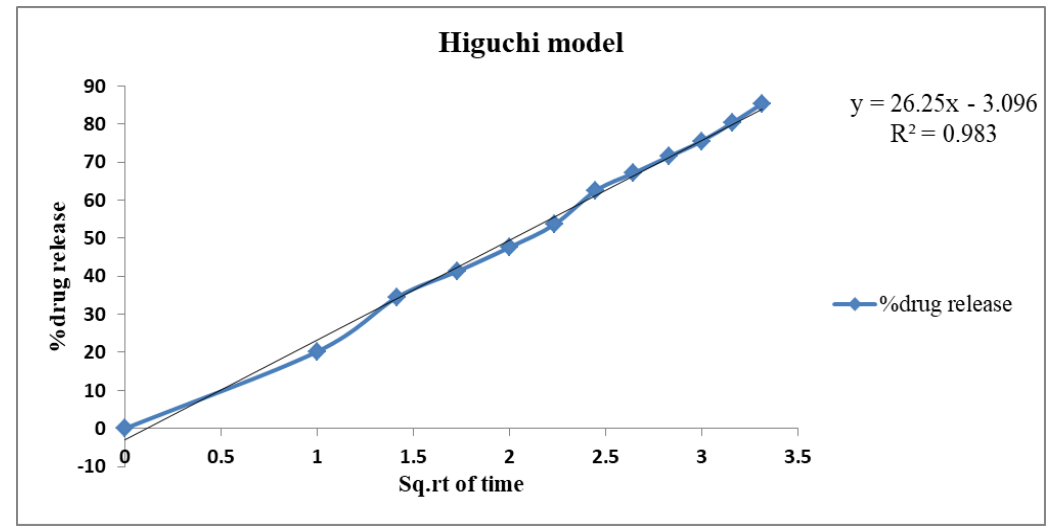

Figure 8 Higuchi's release model 


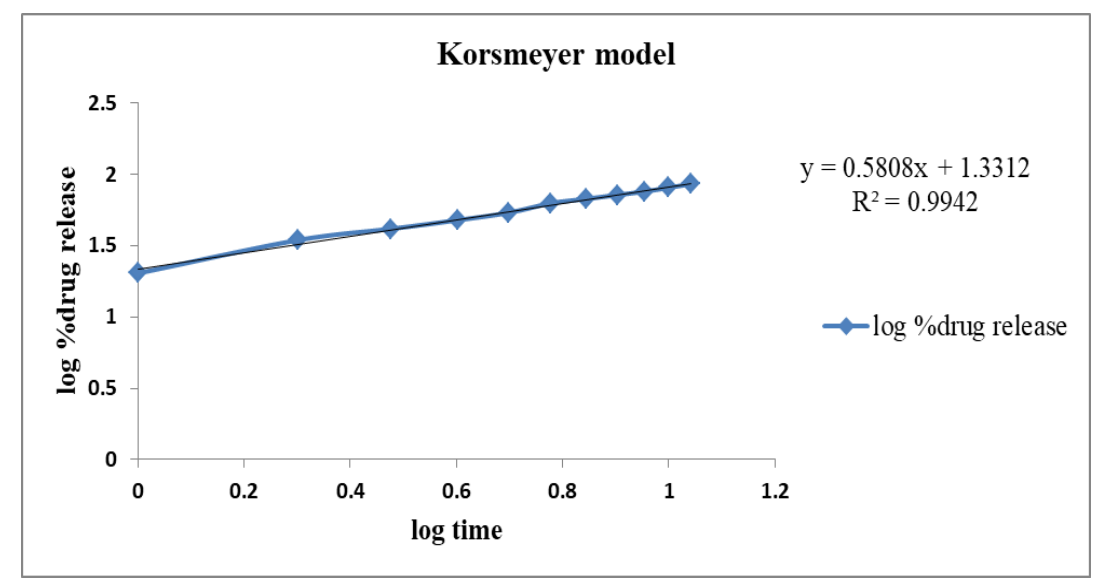

Figure 9 Korsemeyer - Peppas release model

Table $3 \mathrm{R}^{2}$ values of all applied models

\begin{tabular}{|l|l|l|l|l|}
\hline Model name & Zero order & First order & Higuchi's model & Korsmeyer- Peppas model \\
\hline $\mathrm{R}^{2}$ value & 0.974 & 0.986 & 0.983 & 0.994 \\
\hline
\end{tabular}

\subsection{Stability studies}

Accelerated stability studies for 12 weeks revealed that the formulations were stable at up to $45^{\circ} \mathrm{C}$. The results showed that niosomes formulation was quite stable at refrigeration and room temperatures as not much leakage of drug was found at these temperatures. Therefore, the selected Atorvastatin calcium niosomes formulations can be stored at either refrigeration or room temperature. The pure drug shows sensitivity to light and moisture. Therefore formulating it into niosomes can solve this problem to a large extent. Stability studies revealed that the niosomes kept at room temperature $\left(\sim 25^{\circ} \mathrm{C}\right)$ and $40^{\circ}-75 \% \mathrm{RH}$ showed the maximum stability. The values of drug content and in-vitro studies were close to that of the initial data with only slight variations suggesting that it has an acceptable shelf life. It should be stored in a cool, dry place. Niosomes formulations of Atorvastatin calcium of batch ANS9 shows good stability at refrigeration and room temperature in comparison to other.

Table 4 Stability studies of batch ANS9

\begin{tabular}{|l|l|l|l|}
\hline \multirow{2}{*}{ Weeks } & \multicolumn{2}{|l|}{ Batch ANS9(Mean \pm S.D, N=5) } \\
\cline { 2 - 4 } & Refrigeration & Room & Oven \\
\hline 0 & 100 & 100 & 100 \\
\hline 1 & $99.94 \pm 0.01$ & $99.94 \pm 0.06$ & $98.85 \pm 0.04$ \\
\hline 3 & $99.8 \pm 0.03$ & $99.8 \pm 0.05$ & $97.95 \pm 0.08$ \\
\hline 6 & $99.76 \pm 0.05$ & $99.73 \pm 0.04$ & $97.74 \pm 0.12$ \\
\hline 9 & $99.74 \pm 0.03$ & $99.7 \pm 0.06$ & $97.36 \pm 0.31$ \\
\hline 12 & $99.62 \pm 0.02$ & $99.60 \pm 0.02$ & $97 \pm 0.28$ \\
\hline
\end{tabular}

\section{Conclusion}

From the study it is concluded that Atorvastatin calcium noisome were prepared by using different surfactants i.e. Span 40, Span 60, Span 80, Span Tween 20, Tween 40 and Tween 60 by thin film hydration method. Non-ionic surfactants 
along with cholesterol is a suitable carrier for the preparation of niosomes of Atorvastatin calcium. On basis of drug content, particle size morphology, in-vitro release and stability studies, it can be concluded that formulation ANS9was an optimum formulation. However, future experiments should explore the suitability of niosomes with wide variety of drugs having designed drawbacks for improved and effective intended therapy. So, that niosomes are represented as promising drug carriers and promising drug delivery module.

\section{Compliance with ethical standards}

\section{Acknowledgments}

The author thanks Mr. Rajneesh Bansal, Chairman, Himalayan Institute of Pharmacy for providing excellent research facilities.

\section{Disclosure of conflict of interest}

All authors declare no conflict interest is exist.

\section{References}

[1] Singh S, Trivedi S, Jain S. Design and development of proniosome based transdermal delivery of ondansetron hydrochloride. Int J Pharm Biol Res 2012; 3: 191-201.

[2] Jain SK, Awasthi AM, Jain NK, Agrawal GP. Calcium silicate based microballoons of repaglinide for gastro-retentive floating drug delivery: Preparation and in-vitro characterization, Journal of Controlled Release. 2005; 107: 300309.

[3] Schreier H, Bouwstra J. Liposomes and niosomes as topical drug carriers: Dermal and transdermal drug delivery. J Control Release. 1994; 30: 1-15.

[4] Venkatraman S, Davar A, Chester A, Kleiner L, Wise DL, An overview of controlled 465465 release systems, Handbook of Pharmaceutical Controlled Release Technology, New York, Marcel Dekker, Inc. 2000; $431-465$.

[5] Jantzen GM, Robinson JR. Sustained and controlled-release drug delivery systems, in Banker GS, Rhodes CT (Eds.) Modern Pharmaceutics, Third Edition, Revised and Expanded, Drugs and The Pharmaceutical Sciences, 72, Marcell Dekker, Inc., New York. 1995; 575-609.

[6] Gadhiya P, Shukla S, Modi D, Bharadia P, A ReviewNiosomes in Targeted Drug Delivery, International Journal for Pharmaceutical Research Scholars. 2012; 2(61).

[7] Qiu Y, Zhang G, Wise DL. Research and Development Aspects of Oral Controlled- Release Dosage Forms, Handbook of Pharmaceutical Controlled Release Technology, New York, Marcel Dekker, Inc. 2000; $465-503$.

[8] Klaus, Florey. Analytical Profiles of Drug Substances, Academic Press, Inc., New York. 2004; 577.

[9] Bullen WW, Miller RA, Hayes RN. Development and validation of a high- performance liquid chromatography tandem mass spectrometry assay for atorvastatin, ortho-hydroxy atorvastatin, and para-hydroxy atorvastatin in human, dog, and rat plasma, Journal of American Society for Mass Spectrometry. 1999; 10: 55-66.

[10] Bauer S, Mwinyi J, Stoeckle A, Gerl off T, Roots I. Quantification of pravastatin in human plasma and urine after solid phase extraction using high performance liquid chromatography with ultraviolet detection, Journal of Chromatography. 2005; 818: 257-262.

[11] Naseera K, Sajeeth CI, Santhi K. Formulation, Optimization And Evaluation Of Matrix Type of Transdermal System Of Simvastatin Using Permeation Enhancers. Int J Curr Pharm Res. 2012; 4(2): 79-87.

[12] Rogerson A, Cummings J, Willmott N, Florence AT. The Distribution of Doxorubicin in Mice Following Administration in Niosomes. J Pharm Pharmacol. 1988; 40(5): 337-342.

[13] Azmin MN, Florence AT, Handjani-Vila RM, Stuart JFB, Vanlerberghe G, Whittaker JS. The effect of non-ionic surfactant vesicle (niosome) entrapment on the absorption and distribution of methotrexate in mice. J. Pharm. Pharmacol. 1985; 37: 237-242.

[14] Yoshioka T, Sternberg B, Moody M, Florence AT. Niosomes from Span surfactants: Relations between structure and form. J. Pharm. Pharmcol. Supp. 1992; 44: 1044. 
[15] Baillie AJ, Coombs GH, Dolan TF. Non-ionic surfactant vesicles, niosomes, as delivery system for the antileishmanial drug, sodium stribogluconate J.Pharm. Pharmacol. 1986; 38: 502-505.

[16] Balasubramaniam A, Kumar VA, Pillai KS. Formulation and in-vivo evaluation of niosome encapsulated daunorubicin hydrochloride. Drug Dev Ind Pharm. 2002; 28: 1181.

[17] Jagtap A, Inamdar D. Study of antiparkinson's activity of plain and niosomal pentoxifylline. Ind. J. Pharm. Sci. 2001; 63(1): 49-54.

[18] Chauhan S, Luorence MJ. The preparation of polyoxyethylene containing non-ionic surfactant. vesicles. J. Pharm. Pharmacol. 1989; 41: 6. 